\title{
Intelligent System for Real-Time Traffic Recommendations
}

\author{
Pedro Oscar Pérez Murueta ${ }^{1}$, César Raúl Cárdenas Pérez ${ }^{1}$, Jorge Adolfo Ramírez \\ Uresti $^{2}$ \\ ${ }^{1}$ Tecnológico de Monterrey Campus Querétaro, Departamento de Sistemas \\ Computacionales, Av. Epigmenio González 500, Querétaro, México \\ ${ }^{2}$ Tecnológico de Monterrey Campus Estado de México, Departamento de Tecnologías de la \\ Información y Computación, Carr. Lago de Guadalupe Km. 3.5, Margarita Maza de Juárez, \\ Estado de México, México \\ \{pperezm, ccardena, juresti\}@itesm.mx
}

\begin{abstract}
Multi-agent systems have proven to be a powerful technology for the development of large-scale distributed systems that have to deal with highly dynamic systems. The traffic and transportation systems have this feature, which makes them an excellent application area of these systems. This paper proposes a multi-agent system able to make recommendations of travel routes in real time. An agent-based hierarchical architecture analyzes the information obtained from users, open Application Programming Interfaces and social networks to get information about traffic incidents and issues, such as construction sites and traffic congestion and approximate the existing traffic flow. This information is given to an agent-based app that, supported on the preferences and needs of the user, presents recommendations of optimal routes.
\end{abstract}

Keywords: Multi-agent System (MAS), Route Guidance, Algorithm, Recommendations.

\section{Introduction}

The current state of transportation systems in the cities, the main objectives of a system of real-time recommendations for drivers and the benefits of its implementation in a multi-agent system is explained below:

\subsection{Present Situation of Transport in the Cities}

The rapid population growth of cities has created great demands on their transport systems. The traffic generated by public and private transport is the cause of many problems that big cities face today. Problems such as air pollution, hazardous noise levels, economic loss, loss of time and global warming, to name a few. For example, it is estimated that the loss of productivity caused by traffic of Mexico City costs about 33 billion dollars annually [1]. 
In order to mitigate the effects of traffic congestion, the governments of many cities held every year significant investments in transportation infrastructure. Mexico City, for example, conducted an investment of 2.5 billion pesos in 2013 in the construction or improvement of new roads [2]. A similar strategy has been developed by the city of Dhaka, capital of Bangladesh, whose government has opted for an increase in its road infrastructure coupled with the creation of rapid urban transport system [3].

Although this is not the only solution that cities that suffer from this problem have tried. In London, since 2003, those who lead by areas of the city with high traffic problems are required to pay a special fee [4]. And there are many innovative solutions, such as high-speed train that passes over the file that is being developed in China [5].

However, the construction of new roads is usually very costly and inefficient. In fact, Braess [6] showed that adding a new path can result in an increase in total travel time. It is also necessary the application of new technologies that increase the efficiency and effectiveness use of the existing structure. This is the main reason why the development of an information system able to present real-time recommendations on best transport routes to drivers is particularly necessary and important.

\subsection{Objective}

Traffic congestion is one of the biggest problems faced by drivers of large cities. It's main reason for delays, loss of time, stress, significant monetary losses, air pollution and hazardous noise levels. But not only affects drivers, also afflicts passengers, pedestrians, cyclists, buses and taxis. Traffic congestion reduces the quality of life of people and should be undertaken seeking an improvement in transport systems.

However, the high cost of the required technologies for Intelligent Transportation Systems (ITS) are for example in sensors, computers, communication, in-vehicle route guidance among other things and it makes them few large cities having these systems.

Our goal is to develop the conceptual framework for an intelligent system for realtime traffic recommendations inexpensive and able to successfully manage the complexity and dynamism of the city traffic. This system will reduce congestion through more efficient and informed use of existing streets and avenues.

\subsection{Implementation using Multi-agent System}

A multi-agent system is an autonomous computer system, in which there are software agents with a certain level of intelligence. This intelligence can vary from a set of roles (and responsibilities) predetermined to intelligent entities capable of learning. The goal of a multi-agent system is to decompose a problem that can be large, complex and dynamic in smaller single agents and are able to cooperate and communicate [7]. 
Since agent-based systems are able to generate high-quality models for large systems, complex and dynamic, as would a system route guidance in real time, are considered as appropriate technology for the kind of system we want to develop [8].

In this paper we propose an intelligent traffic information system based on agents (MAS) that is able to generate recommendations for alternate routes with less traffic flow in real time considering user preferences. The following section describes the problem to solve. Section 3 describes the framework we have developed for the traffic information system based on real-time multi-agent systems and the agents involved. Finally, Section 4 concludes the paper and provides the future work.

\section{Problem to Solve}

One of the technologies that promise a solution to the problem of congestion are ITSs. An ITS, is a system that integrates communication systems, computers and sensor technology and that enables effective and efficient management of traffic systems [9]. In fact, there are many proposals that show how the use of these systems can reduce travel time for travelers who are still unfamiliar with a given route [10].

However, the high cost of these systems makes that few cities can have them. That's the reason why our proposal, Intelligent System for Real-Time Traffic Recommendations, is based primarily in two technologies that enable us to develop a decentralized ITS at a very low cost. First, the technology of smart phones that have communication systems and sensors to know the location and speed of a user in real time. And second, multi-agent systems that allow us to handle the complexity and dynamism of vehicular traffic. Figure 1 shows the proposed multi-agent architecture. It shows how different agents interact in order to estimate the traffic on the streets and to get real-time recommendations of routes with better flow. 


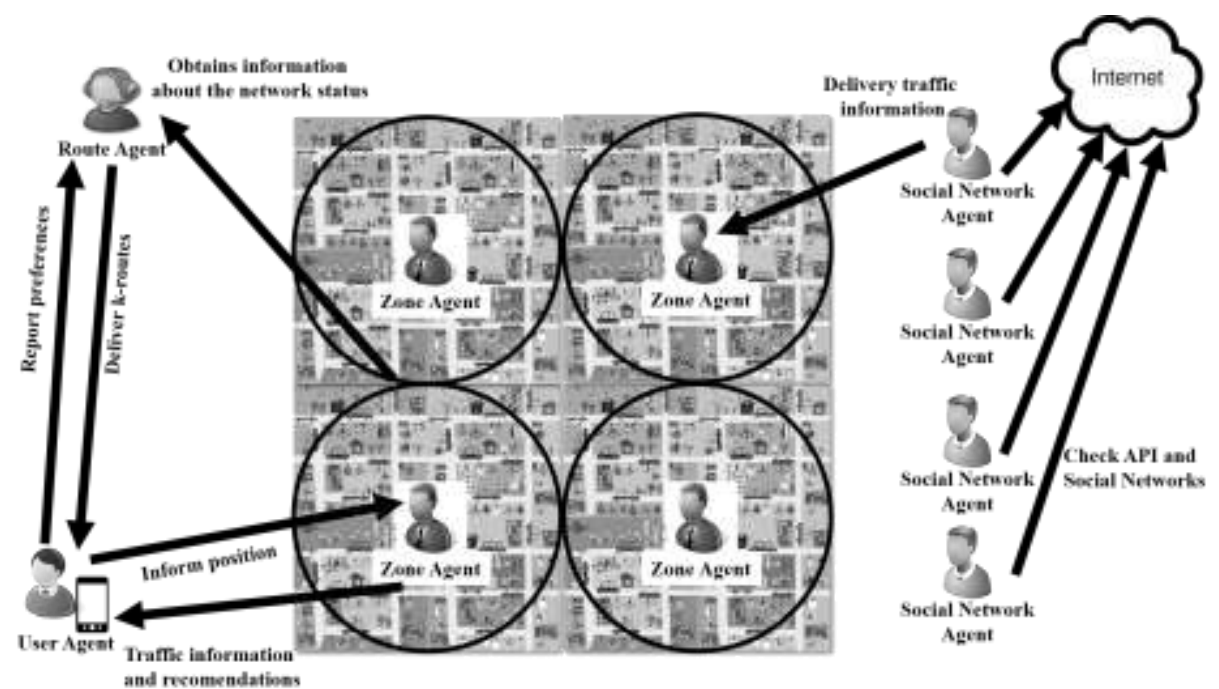

Fig. 1. Different agents interact in order to estimate the traffic on the streets and to get real-time recommendations of routes with better flow.

\section{Framework of System}

After a review of the literature on such systems, we found that a framework composed of three layers was the most suitable for the type of systems that we want to develop [7]. The first layer is an agent platform; in the next layer we find the multiagent architecture and finally the traffic information system. See Figure 2.

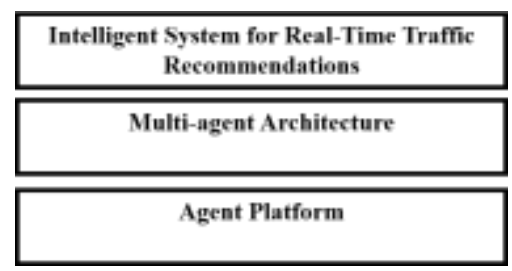

Fig. 2. System Agent Framework.

For the agent platform we use JADE. JADE (Java Agent Development Framework) is a framework based on Java for the development of distributed multiagent applications. It is actually a middleware that provides a set of easy-to-use services and complies with the FIPA standard. Moreover, JADE is very flexible framework and can be used on devices with limited resources such as smart phones [11].

In the multi-agent architecture layer agents and structures necessary for the implementation of traffic information system are located. There are 4 types of agents as shown in Figure 1: User Agent, who represents to the user in the interactions with 
the system; Zone Agent, who is responsible for determining the traffic flow on a set of streets; Social Network Agent, who is monitoring social networks and open traffic Application Programming Interfaces (APIs) looking for information on traffic problems; and Route Agent, who is responsible for generating possible alternative routes.

Finally, we see the implementation of the Intelligent System for Real-Time Traffic Recommendations. Using this scheme, we can extend and deploy new agents to add new functionalities in the system.

Below we describe the main agents that are present in the architecture used for intelligent system of recommendations in real time.

\subsection{User Agent}

This agent represents the user in their interactions with the system. It is responsible for reporting the geographical position of the user. It provides communication and interoperability between the user and the system. It presents alternate routes with better traffic flow based on user preferences. It learns how the user drives in order to better understanding of the preferences thereof. It is responsible for storing previously used routes.

\subsection{Zone Agent}

The zone agent gets information from user agents to determine the density of traffic flow, updating this information in an Origen-Destination (OD) matrix. It informs to the user agents inside its area about possible congestion on the routes that the agents are using. When congestion on its area reaches a threshold, communicate this information to other zone agents.

\subsection{Route Agent}

The route agent is responsible for generating the best $\mathrm{k}$ routes between a given source and destination using preferences sent by the user agent. It stores the results in order to avoid generating a route previously calculated again.

\subsection{Social Network Agent}

The social network agent gets information about traffic incidents and issues, such as construction sites and traffic congestion from open traffic APIs. It analyzes trafficrelated information that may exist in social networks. The information obtained is sent to area agents. 


\section{Conclusions and Future Work}

Although the ITS are systems that allow efficient use of existing traffic infrastructure, the high costs associated with the technologies that are used cause little used in large cities. However, mobile technologies, coupled with the multi-agent systems, make it possible to develop cheaper and easily implemented solutions.

In this paper we present an architecture of an intelligent agent-based system able to know the status of the network traffic and make recommendations, in real time, to users of it. Future work will consist on implement the architecture then to evaluate it.

\section{References}

1. Redacción Atracción 360 (2013) Caos vial frena competitividad en el DF. http://www.atraccion360.com/caos-vial-frena-competitividad-en-el-df. Accessed 3 Sep 2013.

2. Morales A. L. M. (2013) El punto crítico. http://www.elpuntocritico.com/noticiasmetropoli/noticias-df/64286-presenta-gdf-a-trav\%C3\%A9s-de-sobse-informe-sobreinversi\%C3\%B3n-en-remoci\%C3\%B3n-y-mejoramiento-vial.html. Accessed 3 Sep 2013.

3. Mahmud, K., Gope, K., \& Chowdhury, S. (2012) Possible Causes \& Solutions for Traffic Jam and their Impact on the Economy of Dhaka City. Journal of Management and Sustainability 2(2): 112-135.

4. Visitlondon.com. (2014) Official visitor guide. http://www.visitlondon.com/travellerinformation/getting-around-london/congestion-charge. Accessed 26 Feb 2014.

5. Wassener, B. (2010) The New York Times Global Business with REUTERS. http://www.nytimes.com/2010/08/18/business/global/18bus.html?_r=0. Accessed 3 Feb 2014.

6. Braess, D., Nagurney, A., \& Wakolbinger, T. (2005). On a Paradox of Traffic Planning. Transportation Science, 39(4), 446-450. doi:10.1287/trsc.1050.0127

7. Jin, X., Itmi, M., \& Abdulrab, H. (2007). A Cooperative Multi-agent System Simulation Model for Urban Traffic Intelligent Control. In Proceedings of the 2007 Summer Computer Simulation Conference (pp. 953-958). San Diego, CA, USA: Society for Computer Simulation International. Retrieved from http://dl.acm.org/citation.cfm?id=1357910.1358059

8. Chen, B., Cheng, H. H., \& Palen, J. (2009). Integrating mobile agent technology with multiagent systems for distributed traffic detection and management systems. Transportation Research Part C: Emerging Technologies, 17(1), 1-10. doi:10.1016/j.trc.2008.04.003

9. Huang, Y. W., Jing, N., \& Rundensteiner, E. A. (1995) Route guidance support in intelligent transportation systems: an encoded path view approach University of Michigan Technical Report

10. Adler, J. L. (2001) Investigating the learning effects of route guidance and traffic advisories on route choice behavior Transportation Research Part C 9(1), 1-14

11. Bordini, R. H., Braubach, L., Dastani, M., El Fallah-Seghrouchni, A., Gomez-Sanz, J. J., Leite, J., Ricci, A. (2006). A survey of programming languages and platforms for multiagent systems. Informatica (Slovenia), 30(1), 33-44. 